\title{
MINIREVIEW
}

\section{Animal mitochondrial DNA polymorphism: a valuable tool for evolutionary studies ${ }^{1}$}

\author{
ZHANG YAPING, HONG LAN, LIMING SHI $^{2}$. \\ Laboratory of Cellular and Molecular Evolution, Kunming \\ Institute of Zoology, Academia Sinica, Kunming 650223, \\ China.
}

Since the mitochondrial DNA (mtDNA) was discovered[1], a great amount of information have been accumulated about its strucutres and functions[2]. The complete nucleotide sequence of the mitochondrial genome has been determined for one individual in each of the following species: mouse (Mus musculus)[3], human (Homo sapiens)[4], cow (Bos taurus)[5], Xenopus laevis[6], fruit fly (Drosophila yacuba)[7], sea urchin (Strongylocentrotus purpuratus)[8], rat (Rattus novegicus)[9], fin whale (Balaenoptera physalus)[10], and harbor seal (Phoca vitulina)[11]. More than 300 species of animals have been studied on mtDNA $^{3}$. In the past 10 years, mtDNA has been a useful and powerful tool in the field of evolutionary biology and taxonomy.

\section{Introduction to animal mtDNA}

The animal mtDNA is a covalent closed circular molecule. Its genomic structure and gene organization are shown in Fig 1 . The mtDNA is a cytoplasmic genome genetically independent of the nuclear DNA[2]. There are 1000-10000 copies of mtDNA molecules in each cell. The molecular size of higher animal mtDNA ranges between 15.7 and 19.5 kilobase $(\mathrm{kb})$. Within an individual organism, they appear homogeneous in nucleotide sequence. Furthermore, the mtDNA is maternal monoclone and does not seem to undergo recombination during inheritance. It evolves 5-10 folds faster than the single copy nuclear DNA dose at the nucleotide sequence level.

All the features render the animal mtDNA easy to extract and simple to analyze. It has become a useful tool for the evolutionary analysis between closely related.

1. This paper was first published in Zoological Research 1992; 13:289-98 in chinese

2. Corresponding author.

3. This present authors have complied a list of about 300 animal species, of which the mtDNA polymorphism have been studied. Readers who are interested to the list and the references are invited to write to the corresponding author for detailed information. 
mtDNA polymorphism of animals

species or populations within a species.

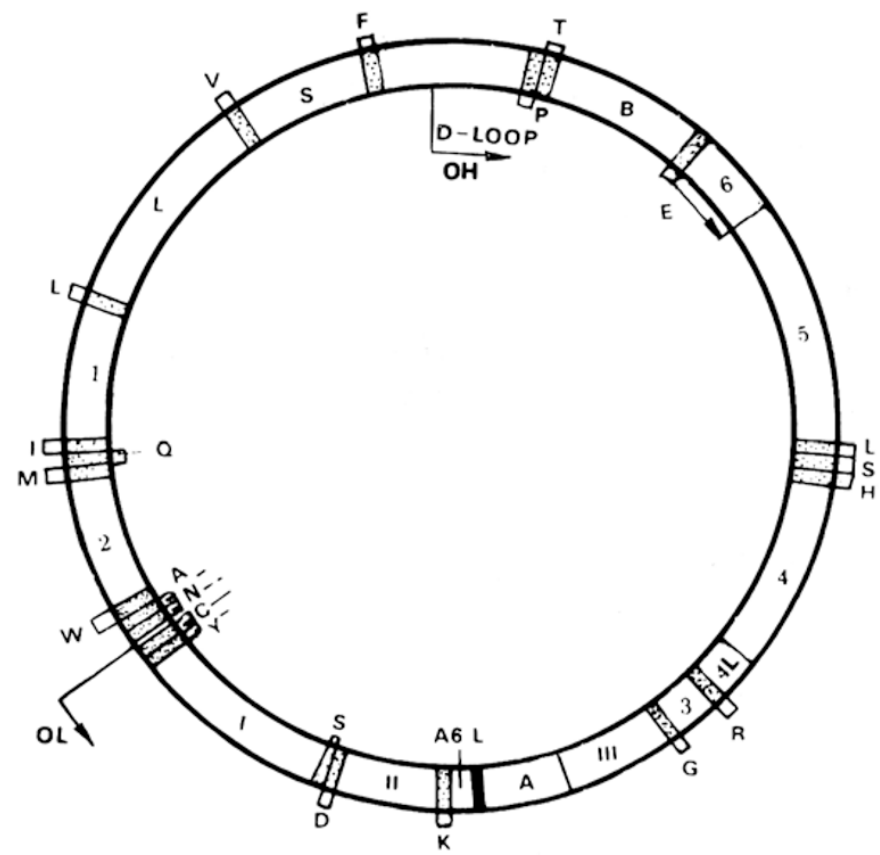

Fig 1. Functional structure of animal mitochondrial DNA (Brown, 1983)

\section{Method for mtDNA polymorphism analysis}

There are 2 major strategies for mtDNA polymorphism analysis. First, the direct comparison on sequences offers an extremely high resolution and yields independent characters that can be converted to estimates of sequence divergence. But this method is time consuming and expensive, hence, it is uncommonly used when analyzing a large number of specimens. Second, the restriction fragments length polymorphism (RFLP) analysis, an alternative method relatively simple and quick for deducing the DNA sequence variation by the comparison of the restriction maps, is usually employed. In the field of evolutionary biology, most people admit that a combination of direct sequencing with RFLP analysis will provide a high-resolution information with high efficiency. In this paper, we focus on the discussion of the mtDNA RFLP.

The standard steps of mtDNA RFLP analysis are as follows:

\section{Experimental manipulations}

1) Extraction of mtDNA. The mtDNA are usually extracted from tissue, blood or cultured cells of animals. 
2) Digestion with restriction endonuclease. Ty pically 15 or more enzymes (yielding more than 30 restriction fragments) are essential for a successful experiment.

3) Electrophoresis of digested fragments. It is often performed in a 0.7-1.2\% agarose gel or a $5 \%$ polyacrylamide gel.

4) Bands visualization. If the amount of mtDN $A$ is very large, we use the ethidium bromide for a detection. However, if the DNA is insufficient, a probe hybridization or an end-labelling technique is usually required.

\section{Data analysis}

Only when the data from RFLP have been converted into genetic distances (revealing the sequence divergence), can an evolutionary relationship between taxa be constructed. There are 2 paths dealing with the conversion from electrophoresis pattern to genetic distances[12].

1) Restriction fragment mathod. The genetic distances are calculated from the proportion of shared fragments. This treatment supposes that if 2 samples share a certain sized fragment, they must also share the flanking cleavage sites. The assumption is generally true for very closely related taxa, but the probabilities of convergence increase as the sequences become more divergent. Therefore, the restriction fragment method is becoming more andmore invalid in phylogenetic analysis,

2) Restriction site method. The genetic distances are calculated from the proportion of shared restriction sites which had been determined on restriction maps. The method overcomes most of the troubles in determining the convergent fragment lengths, despite that the sites themselves may be convergent. Phylogenetic relationship from site data is more reliable, even though mapping the sites will be a little strenuous. Nowadays, only those papers that obtain data from site comparison is acceptable internationally.

\section{Phylogenetic reconstruction}

For a long time, there have been 2 major strategies commonly used for clustering, the un-weighted pair-group (UPG) clustering method[13], and the neighbor joining ( $\mathrm{N} \mathrm{J}$ ) method[14]. The former is more understandable while the latter more accurate in details. A combination of these 2 methods is recommended for constructing the molecular dendrogram from the genetic distance matrix.

However, at present, scientists have recognized that information from the discrete characters is more reliable. The characters axe independent from each other. Each of them is treated separately in certain computational algorithm, out of which the parsimony method and the maximum likelihood method are the most effective ones. By using these methodS, that phylogenetic tree that reflects the evolutionary.relationship among taxa can be constructed. This approach is called "Numeral Cladistics”[15]. 
mtDNA polymorphism of animals

\section{Improvements in the methods of mtDNA RFLP analysis}

A large amount of fresh or frozen tissue $(>5 \mathrm{~g})$ is generally required for mtDNA RFLP analysis, which has limited the utilization of mtDNA in a large scale.

In 1984, Higuchi et al.[16] extracted DNA from the dry skin specimen of quagga (Equus quagga) which was extinct on the earth for more than 100 years. They cloned a fragment and successfully determined the sequence. This pioneer work enlightened us that dead animal specimens stored in the museums are usable, and therefore, is comparable with living animals.

Recently, with the discovery of the polymerase chain reaction (PCR) technique, it is possible to amplify and analyze the DNA extracted from dry specimens (such as the dry pelt and mummy), extremely minute amount of tissue (such as hair follicular cells), or even fossils[17]. The technique of DNA amplification and sequencing enables us to analyze a large number of DNA samples quickly[18]. A combination of PCR with sequencing for dried or even fossil tissues is becoming one of the frontiers of mtDNA evolutionary study.

\section{Applications of mtDNA polymorphism in evolutionary biology and taxonomy}

In this passage, we present examples to illustrate the utilities of mtDNA polymorphism in various fields of evolutionary biology and taxonomy.

\section{Species origination and genetic differentiation}

A great quantity of information has been accumulated, in which the origin and differentiation of the human being is the most notable. Ferris et al[19] investigated the mtDNA from humans and 5 species of apes to examine their phylogenetic interrelationships. In the evolutionary tree, the positions of gibbon and orangutan were quite clear, but the relationship among human, chimpanzee and gorilla remains unclarified. The later sequence data from Hixson and Brown[20] suggested that the human seems to be closer to chimpanzee than to gorilla.

Brown[21] used mtDNA RFLP technique to analyze the polymorphism among human populations. He found that there were wide variations existed in the human mtDNA.

The relationship among different nationalities can also be informed from the comparison of mtDNA RFLPs. For example, early anthropologists thought that the Japanese people were of 2 different origins. The Ainu nationality, living in the northern part of Japan, belonged to the Caucasian race. However, the mtDNA data showed that the Ainus did not possess any peculiar types of mtDNA that exist in the Caucasian people, and that the mtDNA types were not very different between Ainu and non-Ainu people. It suggests that the Ainu people are closer to Mongoloid people rather than to the Caucasians[22].

As regard to geographic origin, the mtDNA data show that the ancestor of modern 
human being is likely to be a female lived in Africa[23], but some authors criticized the method of data analysis[24]. There are also some people supporting an oriental origin[25].

Besides the studies on humans, the origin and differentiation of domestic animals are also interesting. MtDNA RFLP analysis showed that the Asian cattle (genus Bos, including Bos frontalis) are of taurus and indicus origins[26], the pigs in southwest China are monophyletic[27].

\section{Intra-and interspecies phylogenetics}

Is the giant panda close to Ursidae (such as the black and the Malaya sun bear) or Procyonidac (such as the lesser panda)? This is a puzzle having been long quarreled throughout the world. Zhang and Shi[28] studied the mtDNA RFLP of these animals. The genetic distance between the giant panda and the lesser panda is 0.177 , while that from the giant panda to the black bear or the sun bear is 0.306 . It implied that the giant panda is likely to be closer phylogenetically to the lesser panda than to other species of bears of the family Ursidae.

With respect to the intraspecies phylogenetic study, the mtDNA RFLP analysis seems to be the most effective method. Gonzalez- Villasenor[29] investigated the mtDNA from 4 populations of Fundulus heteroclitus. A total of 17 restriction types were detected from 48 individuals. The 17 patterns found can be assorted into 4 groups, corresponding to the 4 populations. The 4 groups further assembled into 2 types, belonging to the 2 subspecies morphologically.

\section{Population genetic structure}

The mtDNA polymorphism is helpful to clarify not only the population structure but also the source of certain structure.

By studying the mtDNA RFLP of the Pacific and Atlantic skyjack tuna (Katsuwonus pelamis), Graves et al.[30] found that the mtDNA polymorphism is great among skyjack tuna, but not limited to a small area. Similar phenomenon was also found in the American eels (Anguilla rostrata)[31]. It was considered that because of their lack of geographic barries in the range of habitat and their high mobility and unusual patterns of life history, the marine organisms carried out a great deal of genetic exchanges among populations so that no significant genetic differentiation could be observed among them.

\section{Animal taxonomy}

By combining the mtDNA polymorphism and the molecular clock, we may calculate the divergence time between taxa and, hence, provide clues to some confusing points in classical taxonomy. For example, in classical taxonomy, the Chinese pheasant (Chrysolopus amherstiae) and the golden pheasant $(C$, pictus) belong to 2 distinct species. However, Zhang and Shi[32] found that the divergence time between these 
mtDNA polymorphism of animals

2 kinds of birds was only 0.6 million years (Myr), which was generally under the rank of subspecies.

In summary, the mtDNA polymorphism is not only a useful genetic marker for studying the relationship of closely related species and populations within a species, but also an effective tool for the investigation of the biodiversity, speciation and evolution.

\section{REFERENCES}

[1] Nass MMK, Nass S. Fibrous structures within the matrix of developing chick embryo mitochondra. Exp Cell Res 1962; 26:427-37.

[2] Brown WM. Evolution of animal mitochondrial DNA. In: Nei M, and Koehn RK eds., Evolution of genes and proteins. Sinauer Associates Inc.: Sunderland. 1983:62-88.

[3] Bibb M J, Van Etten RA, Wright CT, Walberg MW, Clayton DA. Sequenced gene organization of mouse mitochdrial DNA. Cell 1981; 26:167-80.

[4] Anderson S, Bankier AT, Barrell BG et al. Sequence and gene organization of the human mitochondrial genome. Nature 1981; 290:457-65.

[5] Anderson S, de Bruijn MHL, Coulson AR, Eperon IC, Sanger F, Young IG. Complete sequence of bovine mitochondrial genome: conserved features of the mammalian mitochondrial genome. J Mol Evol 1982; 156:683-717.

[6] Roe BA, Ma DP, Wilson RK, Wong JFH. The complete nucleotide sequence of the Xenopus laevis mitochondrial genome. J Biol Chem 1985; 260:9759-74.

[7] Clary DO, Wolstenholme DR. The mitochondrial DNA molecules of Drosophila yacuba: nucleotide sequence, gene organization and genetic code. J Mol Evol 1985; 22:252-72.

[8] Jacobs HT, Elliott D J, Math VB, Farquharson A. Nucleotide sequence and gene organization of sea urchin mitochondrial DNA. J Mol Biol 1988; 202:185-217.

[9] Gadaleta G, Pepe G, De Candia G, Quagliariello C, Sibisa E, Saccone C. The complete nucleotide sequence of the Rattus norvegicus mitochondrial genome: erotic signals revealed by comparative analysis between vertebrates. J Mol Evol 1989; 28:497-516.

[10] Arnason V, Gullberg A, Widegren B. The complete nucleotide sequence of the mitochodrial DNA of the fin whale, Balenoptera physalus. J Mol Evol 1991; 33:556-68.

[11] Arnason V, Johnsson E. The complete mitochondrial DNA sequence of the harbor seal, Phoca vitulina. J Mol Evol 1992; 34:493-505.

[12] Nei M, Li WH. Mathematical model for studying genetic distance in terms of restriction endonucleases. PNAS USA 1979; 76:5269-73.

[13] Sheath PHA, Sokal RR. Numerical taxonomy. San Francisco. 1973.

[14] Saitou N, Nei M. The neighbor-joining method: a new method for restriction phylogenetic tree. Mol Biol Evol 1987; 4:406-25.

[15] Hillis DM, Moritz C. Molecular Systematics. Sinauer Associates Inc: Sunderland, USA. 1990.

[16 Higuchi R, Bowman B, Freiberger M, Ryder OA, Wilson A C. DNA sequence from the quagga, an extinct member of horse family. Nature 1984; 312:281-4.

[17] Pääbo s. Ancient DNA: extraction, characterization, molecular cloning and enzymatic amplification. PNAS USA 1989; 86:1939-43.

[18] Thomas WK, Pääbo S, Villabance FX, Wilson AC. Spatial and temporal continuity of Kangaroo rat populations shown by sequencing mitochondrial DNA from museum specimens. J Mol Evol 1990; 31:101-21.

[19] Ferris SD, Brown WM, Davidson WS, Wilson AC. Extensive polymorphism in the mitochondrial DNA of apes. PNAS USA 1981; 78:6319-23. 
[20] Hixson JE, Brown WM. A comparison of the small ribosomal RNA genes from mitochondrial DNA of the great apes and humans: sequence, structure, evolution, and phylogenetic implications. Mol Biol Evol 1986; 3:1-18.

[21]Brown WM. Polymorphism in mitochondrial DNA of Humans as revealed by restriction endonuclease analysis. PNAS USA 1980; 77:3605-9.

[22] Harihara S, Saitou MH, Hirai M et al. Mitochondrial DNA polymorphism in Japanese living in Hollaido. Jpn J Hum Genet 1986; 31:73-83.

[23]Cann RL. DNA and human origins. Am Rev Anthrop 1988; 17:127-43.

[24]Maddison DR, Ruvolo M, Swofford DL. Geographic origins of human mitochondrial DNA: phylogenetic evidence from control region sequences. Syst Biol 1992; 41:111-24.

[25]Yu MS, Qiu XF, Xue JL et al. Study of mitochondrial DNA polymorphism of Chinese people . Zhong Guo Ke Xue(Chinese) 1988; (1):60-70.

[26] Bhat PP, Mishra BP, Bhat PN. Polymorphism of mitochondrial DNA (mtDNA) in cattle and buffalo. Biochem Genet 1990; 28:311-8.

[27]Lan H, Shi LM. Origin and genetic differentiation of native pigs in Yunnan province:an approach from the mitochondrial DNA polymorphism. Biochem Genet 1993; 31:51-60.

[28]Zhang YP, Shi LM. Riddle of the giant panda. Nature 1991a: 352:574.

[29] Gonzalez-Villasenor LI, Powers D. Mitochondrial DNA restriction site polymorphisms in the teleost Fundulus heteroclitus support secondary integradation. Evolution 1990; 44:27-37.

[30]Graves JE, Ferris SD, Dizon AE. Close genetic similarity of Atlantic and Pacific skyjack tuna (Katsuwonus pelamis) demonstrated with restriction endonuclease analysis of mitchondrial DNA. Mar Biol 1984; 79:315-9.

[31]Avise JC, Helfman GS, Saunder NC, Halse LS. Mitochondrial DNA differentiation in North Atlantic eels: population genetic consequence of an unusual life history pattern. PNAS USA 1986; 83:4350-3.

[32]Zhang YP, Shi LM. Comparative study of mitochondrial DNA from pheasant. Zoo Res(Chinese) 1991b; 12:387-92.

Received 28-3-1992. Revised 31-5-1993. Accepted 9-6-1993 